\title{
Cultura sobre maternidad y paternidad y su repercusión en la concepción de la infertilidad
}

\author{
Culture on motherhood and fatherhood and its impact on the \\ concept of infertility
}

\author{
Zoe Díaz Bernal I; Dailys García Jordá ${ }^{\text {II }}$

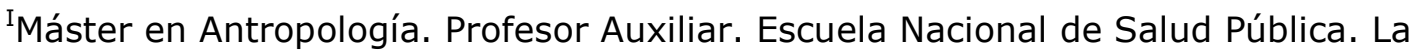 \\ Habana, Cuba. \\ II Máster en Antropología. Investigador Agregado. Facultad de Biología, Universidad \\ de La Habana. La Habana, Cuba.
}

\section{RESUMEN}

Introducción La infertilidad es el hecho que una vez vivenciado provoca la reformulación de las expectativas en relación con la maternidad y la paternidad.

Objetivos Explorar la cultura existente en Cuba en relación con la maternidad y la paternidad, su repercusión en la concepción de la infertilidad y la manera en que esta es percibida por el individuo y la colectividad.

Métodos Se realizaron entrevistas en profundidad a nueve expertos en reproducción humana y salud pública, seleccionados por un muestreo en cascada a partir de un informante clave. El número de entrevistas se definió según el criterio de "saturación" de la información.

Resultados Se identificaron tres etapas: Cuba pre-revolucionaria, década del 60 y los últimos 20 años. Todos los entrevistados coincidieron en que en la actualidad el binomio mujer-madre persiste, solo ha cambiado lo culturalmente añadido que hace que la mujer razone cuándo es más conveniente ser madre y si desea tener un hijo o dos a lo sumo, lo que está tornando crítica la situación en cuanto al reemplazo poblacional. En relación con los hombres infértiles, dos expertos plantearon la descalificación y el cuestionamiento que sobre su virilidad hace la sociedad cubana. Se obtuvo escasa información referida a la percepción de la infertilidad por la pareja infértil y por la colectividad.

Conclusiones Dentro del patrón sociocultural cubano, se reconoce a la mujer como principal responsable de la decisión de tener o no descendencia y de su cantidad. Lo anterior repercute en la concepción de la infertilidad, desde su atención médica regulada con exclusión de las vivencias de las parejas y la prácticamente ausencia 
del varón a los servicios de atención, hasta la poca información que se tiene sobre lo que experimentan los hombres, la pareja infértil y la familia cubana ante la infecundidad.

Palabras clave: Salud reproductiva, infertilidad, entrevistas en profundidad, investigación cualitativa.

\section{ABSTRACT}

Introduction Infertility is an event that whenever it occurs, it causes the reshaping of the expectations about motherhood and fatherhood.

Objectives To explore the existing culture in Cuba about motherhood and fatherhood and its impact on the concept of infertility, as well as to know how this is perceived by the individual and the community.

Methods In-depth interviews were administered to 9 experts in human reproduction and public health, who were selected through a cascade-sampling from a key informant. The number of interviews was defined according to the "saturation" criterion" of the information received.

Results The first result was the identification of three stages: Cuba before the revolution, the 60 's and the last 20 years up to the present. All the interviewed experts agreed that the woman-mother binomial persisted, it was just that the level of culture had increased, which made the woman decide the most suitable time for her to become a mother and the number of children -one child or two at mostleading to a critical situation in terms of the natural replacement of the Cuban population. Regarding infertile men, two experts pointed out that their virility is generally questioned by the Cuban society. Limited information on the perception of infertility by the infertile couple and the community as well was obtained.

Conclusions Within the social-cultural pattern of the Cuban society, it is recognized that the woman is the responsible for making a decision of having offspring or not and of the number of children. The above-mentioned has an impact on the concept of infertility, starting from the controlled medical care with the couple's experience being excluded and the man being practically absent to the medical care appointments, to the limited information available regarding what the men, the infertile couple and the Cuban family feel in case of infertility.

Key words: Reproductive health, infertility, in-depth interviews, qualitative research.

\section{INTRODUCCIÓN}

El culto a la fertilidad es algo tan antiguo como lo es la existencia humana y ha supuesto gran importancia para la supervivencia de la humanidad. La infertilidad se ha llegado a considerar de muy diversas maneras: como deshonra, como inferioridad, como maldición o castigo de dioses, e incluso en Egipto, se consideró como causa para la disolución del matrimonio. Por lo tanto, la preocupación por la falta de descendencia no es un tema actual, como tampoco lo son sus consecuencias y sus tratamientos. Así, desde la antigüedad el deseo de 
descendencia ha sido muy fuerte y se han buscado remedios de todos los tipos. Los problemas de fertilidad tienen una gran importancia tanto familiar como socialmente desde siempre. ${ }^{1}$

La infertilidad es precisamente el hecho, ya sea sospechado, verazmente diagnosticado o no, que una vez vivenciado provoca la reformulación de las expectativas en relación con la maternidad y la paternidad. Hasta ese punto, casi la totalidad de las personas ven como algo natural y hasta fisiológico, lograr tener descendencia. $^{2}$

La procreación y el tener descendencia tienen significados diferentes para cada uno de los miembros de la pareja. Para las mujeres suele ser sinónimo de desarrollo de su función materna, de protección, afecto y educación, mientras para los varones tiende a tener un significado más dirigido a sentimientos de poder, de patriarcado, de protección, de proveedor, de arribo a la plenitud de la hombría, como ente eminentemente masculino. ${ }^{3}$ Más allá de la reproducción biológica, son la reproducción social y los espacios donde esta se desarrolla, los que determinan en última instancia las percepciones individuales y colectivas de la función reproductiva y la infertilidad.

Esta investigación pretende ser el inicio de un proceso investigativo mucho más abarcador y holístico, que explique y haga comprender los significados y significantes que la infertilidad tiene para las personas, en los diferentes niveles de interacción sociocultural, de hecho, se pretende explorar cuál ha sido la cultura en Cuba en relación con la maternidad y la paternidad y su correspondencia con la concepción de la infertilidad, así como la manera en que esta ha sido percibida por el individuo y la colectividad.

\section{MÉTODOS}

Se realizaron entrevistas en profundidad a nueve expertos en Reproducción Humana y Salud Pública, la mayoría de ellos médicos, especialistas en ginecoobstetricia, con 30 años o más de servicio. Seis de ellos son expertos en el área de ginecoobstetricia, dos en atención a la infertilidad y uno en salud pública, seleccionados por un muestreo en cascada a partir de un informante clave (médica, ginecoobstetra, Profesor Consultante, mayor de 70 años, con 40 años de Servicio, residente en La Habana). Se analizó el contenido de las entrevistas.

La mayoría de los expertos entrevistados estudiaban la carrera de medicina o comenzaron a ejercer su profesión durante la primera década posterior al triunfo de la Revolución en 1959. Durante los años de estudios se encontraban vinculados a la asistencia médica y una vez graduados cumplieron el servicio médico rural, por lo que poseen conocimientos sobre la realidad cubana y en particular de la situación de salud, urbana y rural.

Las entrevistas en profundidad, según criterio de Dingwall $R$, citado por Callejo Gallego $J_{1}^{4}$ permiten la aproximación a la experiencia vivida por los sujetos en relación con el tema investigado. El número de entrevistas se definió según el criterio de "saturación" de la información, que se utiliza en la recolección de datos en la investigación cualitativa y refiere, que se deja de entrevistar en el momento en que el dato ya no aporta nada nuevo y la información suele hacerse repetitiva, ${ }^{5}$ lo que en otras palabras sería dejar de entrevistar cuando se ha obtenido suficiente material para la comparación y corroboración mutua de la información obtenida, que irremediablemente será fragmentaria en algunos aspectos. ${ }^{4}$ Las entrevistas 
fueron grabadas, previo consentimiento de los participantes, se transcribieron y se tomaron notas relacionadas con el componente extraverbal observado (contacto visual, gestos faciales, gesticulación, tono de voz y otros). Durante las entrevistas se crearon las condiciones para establecer la mejor empatía posible, se respetó el protagonismo de los/as entrevistados/as y se preservó su identidad.

Las principales áreas exploradas durante las entrevistas fueron, en primera instancia, la concepción de la maternidad y la paternidad, eje a partir del cual podría estructurarse y comprenderse la concepción de la infertilidad, referida a la cultura y percepción colectiva e individual, sobre la infertilidad como evento y condición.

\section{RESULTADOS}

Un primer resultado aportado por esta investigación fue la identificación de tres etapas, dentro de las cuales se definieron hitos, entendidos estos como aquellos sucesos externos e internos narrados por el entrevistado, que son presentados como cruciales en el curso de su vida, aquellos momentos claves a los cuales se les otorga capacidad explicativa, explícita e implícitamente. ${ }^{6}$

Etapas:

1. Previo al año 1959: Cuba pre-revolucionaria.

2. Década del 60.

3. Últimos 20 años.

La caracterización de dichas etapas habla sobre todo de la cultura que sobre la maternidad y la paternidad existía en Cuba en cada una de ellas, lo que parece trascendental y punto de partida para los análisis que se hacen posteriormente en cuanto a la incapacidad para procrear de mujeres y hombres en el contexto cubano.

\section{Caracterización de la etapa 1}

La totalidad de los entrevistados coincidió en apuntar que en esta etapa, el sentimiento maternal en la mujer, entendido como el deseo o inclinación hacia la maternidad, es un sentimiento instintivo. No así para el hombre el sentimiento paternal, quien después de haber tenido una vida sexual más o menos activa, va al matrimonio en busca de hijos y formar una familia. Muy aparejado a ello, e igualmente de carácter consensual entre los entrevistados, primaba entre hombres y mujeres la concebida igualdad matemática de mujer= esposa = madre, tanto a nivel colectivo como individual. También fue criterio unánime que en dicha etapa las conductas sexuales de varones eran mucho más liberales y desprejuiciadas que las de las mujeres, pues la presión social para ellos era mucho menor que para las féminas.

Una característica identificada unánimemente fue que, desde entonces, la cultura reproductiva en Cuba era abortiva y no anticonceptiva y aún cuando la práctica del aborto era ilegal, se realizaba con grandes éxitos, reconocido internacionalmente y así mismo demandado. 
Las jóvenes venían desde los Estados Unidos a hacerse abortos en consultas particulares...conocí muchos médicos que vivían muy bien de eso...eran muy rentables esas consultas (sic) (E8)*.

Según estos expertos, el uso del condón por las mujeres, aunque escaso, estaba mayormente asociado al método anticonceptivo y no para evitar el contagio de infección de transmisión sexual (ITS), era bastante rechazado por los hombres, quienes entonces se arriesgaban a contraer un grupo importante de estas enfermedades con bastante frecuencia. No obstante, y aunque tampoco la anticoncepción era privilegiada por la práctica en pareja, era bastante normal encontrar mujeres multíparas dado que la concepción de una extensa prole era bien vista, aceptada y hasta considerada una necesidad en la sociedad cubana prerevolucionaria.

Aunque los expertos no pudieron opinar acerca de la incidencia y prevalencia de la infertilidad en esta etapa, reconocieron que para entonces, como para la actualidad, no se conocían las tasas de prevalencia de la infertilidad, por lo que no puede afirmarse que no constituyera un problema de salud.

\section{Caracterización de la etapa 2}

Como se conoce, esta etapa se caracterizó por la generación de numerosos y profundos cambios a todos los niveles de la sociedad. A raíz del triunfo revolucionario la nueva dirección del país comenzó a poner en práctica mucho, y más, de la plataforma programática del Moncada, que por supuesto incluía la atención y cuidado de la salud del pueblo. Para entonces los indicadores de salud que exhibía Cuba eran para nada halagüeños, la situación de salud de la población rural era deplorable y a todo ello se adicionaba la deserción y emigración o ambas, de médicos formados en la etapa pre-revolucionaria con fuerte arraigo pequeño burgués. Es precisamente el Triunfo de la Revolución cubana el primer hito reconocible en esta investigación.

A pesar de que en esta etapa la mujer tuvo una participación activa en las tareas de construcción de una nueva sociedad, para lo cual comenzó a insertarse cada vez más en los espacios laborales, de enseñanza y hasta políticos, los entrevistados coincidieron en plantear que persistía el deseo por parte de ellas y de las parejas constituidas, de tener descendencia, y la idea de una extensa prole no era desechada y hasta deseada. De esta manera los expertos entrevistados, para los que la experiencia del servicio posgraduado en zonas rurales les permitió palpar la realidad en lugares distantes e inaccesibles de la geografía cubana, testimoniaron la relativa facilidad de hallar mujeres-madres multíparas sobre todo en la zona oriental del país.

Los entrevistados identificaron que ya en esta época se prestaban algunos servicios de infertilidad, que aunque eran escasos por la precariedad de especialistas, acudían las mujeres que no lograban concebir, las que iban casi siempre solas. Puede apreciarse que ya entonces la población exhibía cierta cultura médica.

Donde yo hice mi postgraduado vi mujeres que venían a parir y que ya tenían seis hijos (sic) (E6).

\section{Caracterización de la etapa 3}

La visión generalizada de los expertos sobre esta etapa es la percepción de un cambio en cuanto a la mentalidad de las mujeres acerca de la maternidad. Ellos apreciaron que si bien sigue siendo instintivo tener descendencia, ese "instinto" es 
razonado, es valorado, de acuerdo a los proyectos de vida y la cultura de cada mujer, según su procedencia sociocultural. A pesar de ello, la mitad de los entrevistados opinaron que quien se concibe mujer se siente obligada a parir y aunque no sea ya puramente instintivo, ante la imposibilidad no se consideran mujeres biológicamente completas. Todos los entrevistados concordaron en que el binomio mujer $=$ madre persiste a pesar del vertiginoso avance en instrucción y desarrollo social que ha tenido la mujer cubana, pero el instinto maternal se mantiene invariable y solo ha cambiado lo culturalmente añadido, que hace que la mujer razone cuándo es más conveniente ser madre. Así, la mayoría de ellos coincidió en apuntar que aunque la mujer sigue deseando ser madre, en la actualidad solo desea tener un hijo y a lo sumo dos, lo que ha tornando crítica la situación en cuanto al reemplazo poblacional en Cuba. Este es el segundo de los hitos identificables a través de las narraciones discursivas de estos expertos.

En una sola generación cambió el patrón de natalidad en Cuba, la mujer pasó de tener una prole extensa a tener descendencia de hasta 3 hijos... icuanto mas! (sic) (E8).

\section{En el contexto cubano}

Llama la atención como la totalidad de los entrevistados en sus discursos, ubica a la mujer como principal responsable de la decisión de tener o no descendencia y de su cantidad, lo que reconocieron como esencia generalizada en el patrón sociocultural cubano. Por otra parte, la mayoría opinó que la maternidad es algo culturalmente aprendido, es una transmisión heredada por la mujer de sus antecesoras, lo que muy bien concuerda con la conceptualización que de procesos bioculturales hacen Vargas y Casillas $^{7}$ para definir aquellos hechos o situaciones, que derivadas de la fisiología humana son modificadas y matizadas por la cultura.

Todo ello de cierta manera condiciona el arribo natural de la mujer a la maternidad, y todo ello, además, incide en el maternaje: modo y concepción de la crianza y relación con la descendencia. Dos expertas vinculadas por muchos años a los servicios de maternidad, coincidieron en remarcar que el sentimiento maternal de la mujer y familias cubanas es muy elevado, lo que condiciona que el abandono de la descendencia sea un evento raro y el apego a ella se sobreexprese ante el fallecimiento del recién nacido, al que consideran parte de la familia, y ello conlleva un profundo dolor al interior de esta.

Aunque fue un criterio que no logró corroborarse con el de otros entrevistados, uno de ellos planteó la relación directamente biológica que tiene la paternidad y la maternidad para los hombres y mujeres cubanos/as, para quienes ser padre y madre es parir. Lo anterior pareció importante consignarlo, por tratarse de un experto que ha estudiado los significados y significantes de la infertilidad en el contexto cubano, a través de las narraciones discursivas de las personas, lo cual ha sido una metodología de investigación poco usada en este campo y como ya se conoce de innegable valor para comprender el por qué de los fenómenos que han sido poco estudiados, como es el caso de la infertilidad.

En tal sentido, a las dos expertas anteriormente mencionadas se le suma otro experto, al apuntar coincidentemente hacia la fuerte presión sociocultural que gravita sobre las mujeres que presentan dificultades para concebir, pues la demanda social desde la construcción de género las obliga a ser madres, ya que persiste en el imaginario y cosmovisión en Cuba, que el concepto de mujer está ligado al de esposa y madre, de ahí que la que no logre ser madre se considera y es considerada, disminuida. Sin embargo, a partir del comentario hecho por la informante clave o primera entrevistada respecto al carácter permisivo que ha ido 
ganando la sociedad cubana, en cuanto a comportamientos sexuales y reproductivos, otros tres expertos reconocieron que la liberación de la mujer en relación con la conquista de espacios anteriormente privativos para los hombres y su inserción en el entorno socioeconómico casi a la par de ellos, ha frenado la maternidad, por implicar un doble esfuerzo para ellas, quienes según el patrón genosocial son aún las principales responsables de la crianza y cuidado de la descendencia.

En tal sentido coincidieron cuatro entrevistados, al referir que la mujer cubana fue priorizando otras actividades por encima de la maternidad ya que esta puede interferir con su desarrollo profesional, lo que explica de alguna manera el fenómeno que se está viendo en la actualidad al recibir en las maternidades mujeres embarazadas con 40 y más años: la maternidad postergada, no demasiado desligada de la infertilidad o disminución de la capacidad reproductiva.

Al ser la sociedad más permisiva han aparecido mujeres que han optado por su libertad...vida libre, y no les interesa ni el matrimonio ni tener hijos. (sic) (E1).

Ya hay mujeres que comprenden que no necesariamente tienen que ser madres (sic) (E6).

La mayoría de los entrevistados identificó otros factores incidentes en el proceso de postergación de la maternidad en la Cuba de hoy: la mujer espera reunir ciertas condiciones materiales para tener descendencia y el logro de estas es muy demorado, el tema de la escasez de vivienda que propicia la convivencia de varias generaciones en espacios reducidos, las dificultades para encontrar pareja y el consecuente desplazamiento de la edad de casamiento, todo lo cual está influyendo en la incidencia de la infertilidad, ya que se posterga la maternidad a costa de la ya reconocida finita reserva ovárica.

La primera entrevistada y otros tres expertos, reconocieron que la liberación de la mujer cubana a partir del triunfo revolucionario había sido vertiginosa, sobre todo en cuanto a su comportamiento sexual, pero que dicho cambio no había sido del todo positivo pues a la mujer le faltaba aún instrucción y educación para asumirla responsablemente y ello influyó en la incidencia de la infertilidad. Cada vez más las mujeres muestran conductas sexuales promiscuas y desprotegidas, que elevan de alguna manera la incidencia de las ITS con las consecuencias anatomofisiológicas esperadas: obstrucciones tubarias, embarazos ectópicos y otras.

La mujer quiere ser igual al hombre, pero al tener otra anatomía más riesgosa, paga muy caro según su conducta sexual: una mujer con absceso tubo-ovárico nunca más podrá tener un embarazo, porque aunque se preserve una de las trompas, ésta siempre queda dañada(sic) (E1).

Antes de darle libertad a la mujer hay que darle educación, crearle el concepto de su libertad y el concepto de la protección de su salud. Faltó un poco de educación para asumir esa liberación de la mujer tan violenta después del triunfo de la Revolución (E1, E2, E3, E8).

Otros tres entrevistados consideraron la liberación de la mujer como aspecto positivo y opinaron que dicha liberación no había sido exclusiva para ellas, también los hombres la experimentaron y eran posiblemente los más promiscuos e irresponsables en cuanto a comportamientos sexuales.

Una vez más las dos expertas citadas arriba, reconocieron entre las actitudes que necesitan de una mayor educación, el uso del condón y la conciliación en cuanto a 
su empleo por parte de los hombres por exigencia de sus parejas. Ellas plantean que en ocasiones es la propia mujer quien se niega a usarlo, y otras justifican a su pareja por negarse a ello, lo cual habla de su autoestima y nivel de educación para la salud. Estas expertas, quienes además trabajan vinculadas a servicios de atención a la infertilidad, reconocieron en ello un factor negativo para el tratamiento de esta incapacidad para tener descendencia y su solución.

...eso incide en el éxito del tratamiento de la infertilidad, pues hemos tenido que suspender varias veces la inseminación de mujeres por ETS y es una situación delicada(sic) (E4).

Una de dichas expertas emitió un razonamiento relevante aún cuando no fue posible corroborarlo entre otros entrevistados, y es que si bien la Revolución conllevó a una explosión de la participación femenina a escala social, dicha realidad se ha modificado últimamente debido a los cambios socioeconómicos experimentados en las últimas décadas. Las mujeres de alguna manera han regresado a ser amas de casas, que esperan por la manutención masculina, y si bien podría suponerse que dichas mujeres tuvieran más descendencia, esto no es del todo probable, pues ellas buscan elevar su estatus económico más que conformar una familia, con los sacrificios propios de la maternidad.

...ya te encuentras un montón de muchachas jóvenes en edad laboral, que están en la casa, esperando que los novios, los maridos, las mantengan, mirando novelas en el DVD, la antena, esa es la vida de ellas... por lo que hay que empezar un nuevo análisis de la mentalidad de la mujer cubana (sic) (E4).

Como se aclaró, uno de los objetivos de la presente investigación es determinar cuál es la percepción de la infertilidad para el individuo y la colectividad, en el contexto cubano. En cuanto a esto, se obtuvo cierta diferenciación en la información referida a los siguientes grupos:

- Para las mujeres cubanas.

- Para los hombres cubanos.

- Para las parejas infértiles.

- Para la colectividad.

\section{Para las mujeres cubanas}

Tres entrevistados expresaron la casi categórica inconformidad de la mujer cubana ante la imposibilidad de procrear, aún cuando con el transcurso de los años logre compensarlo con otros aspectos de la vida. En tal sentido plantearon que la mujer se considera entonces a sí misma como disminuida. Otra vez vuelve a argumentarse el sentimiento de pérdida, de duelo, con el carácter instintivo de la maternidad, lo que es algo que debe ser estudiado con profundidad, pues que no sorprenda que como se plantea en la introducción del presente artículo, también sea esta concepción instintiva, un constructo sociocultural.

La mayoría de las pacientes caen en estado depresivo cuando menstrúan, de pasarse 3 días en cama, de no bañarse, no hablarle a los maridos, de llorar, estados depresivos de verdad. De no querer vestirse, de no ir a trabajar, son mujeres que están emocionalmente muy golpeadas (sic) (E4). 
Resultó criterio compartido por la totalidad de los expertos, que la infertilidad incide en el desarrollo personal, familiar y profesional de las mujeres que la padecen, lo que entorpece su avance y aporta elementos a la disfuncionalidad de sus relaciones de pareja y familiares y ponen incluso en riesgo las primeras.

\section{Para los hombres cubanos}

Dos de los expertos fueron esencialmente los más informativos en cuanto a la percepción de la infertilidad para los hombres. Dichos expertos insistieron en plantear la descalificación y el cuestionamiento sobre su virilidad, que perciben los hombres infértiles por parte de la sociedad cubana. Uno de ellos, estudioso del tema de la infertilidad en la población masculina cubana, remarcó el sufrimiento del hombre infértil más desde la demanda social que desde el propio deseo de ser padre, elementos que deben retomarse en próximas investigaciones para intentar establecer en lo posible, las explicaciones que desde lo sociocultural pueda tener tal percepción. Este mismo experto aportó individualmente la idea de la construcción del padecer de la infertilidad para los hombres a una edad bien temprana, incluso desde la adolescencia, cuando aún no desea tener descendencia pero desea comprobar si será capaz de embarazar a una mujer en el futuro. Otro aspecto que merece ser explorado.

Cuando se le dice a un hombre que no produce espermatozoides y que no tiene solución, eso es lo más duro que hay. Pues el hombre no está preparado para llorar, lo criaron con la cosa aquella de que el hombre no llora y se les salen las lágrimas. Al más de los mejores se le salen las lágrimas, porque es muy difícil. El hombre llora como un muchacho chiquito, eso es doloroso (sic) (E4).

\section{Para las parejas infértiles}

Aunque fue escasa la información referida a la pareja, lo planteado por dos expertos sobre la percepción de la infertilidad por la pareja cubana, coincidió con lo referido en la literatura publicada sobre el tema a nivel internacional para otros contextos, y que habla de las crisis de la pareja con peligro incluso para la disolución del vínculo. $1,8,9$

\section{Para la colectividad}

Si bien la totalidad de los expertos coincidió en apuntar que la infertilidad es un evento inesperado, el criterio de uno de ellos refiere que en la actualidad se acepta cada vez más que no todas las parejas pueden tener descendencia, criterio que no logró ser corroborado a través del análisis discursivo del resto de los entrevistados. Sin embargo, para la mayoría, la infertilidad, desde el punto de vista de la colectividad, es o una enfermedad o un problema de salud que requiere ser tratado para ser resuelto.

\section{El entramado de creencias, actitudes y prácticas}

Sobre la creencia bastante extendida en la población de que la infertilidad se debe a condiciones femeninas, la mayoría de los expertos afirmó que obedece al machismo que con fuerte arraigo prevalece en la cultura cubana. Fue consenso que el pensamiento de los hombres cubanos no ha evolucionado como el de las mujeres, lo que queda reflejado en la resistencia a asistir a las consultas, a realizarse pruebas diagnósticas como el espermiograma e incluso en el acto de vanagloriarse ante su resultado satisfactorio, como fue expresado por tres expertos. 
Los entrevistados coincidieron en que es la medicina alopática la primera instancia a la que acuden las personas ante la sospecha de infertilidad, resultado que se aviene con los de otras investigaciones realizadas en Cuba. ${ }^{2}$

Por otra parte, la mayoría da fe de que muchas mujeres emplean en algún momento métodos alternativos, aún sin consultarlo con su médico, que en muchos casos tienen acciones y efectos contradictorios a los diagnósticos y tratamientos. Una vez más, las dos expertas anteriormente destacadas, de las que se insiste en decir que se encuentran directamente vinculadas a la atención de parejas infértiles, reconocieron que habían sido consultadas al respecto y después de evaluar y explicar las posibles contraindicaciones de los métodos a emplear, aprobaron casi siempre que sus pacientes los asumieran. Ambas expertas alegaron que dados los sentimientos de culpa que suelen gravitar sobre las mujeres, el no acudir a estos métodos y no lograr en definitiva el tan añorado embarazo, puede contribuir al daño psicológico de ellas y de sus parejas.

\section{CONSIDERACIONES FINALES}

Se apreció que las entrevistas realizadas ofrecieron poca información sobre la percepción de la infertilidad para quienes la padecen, en especial cuando se hizo referencia a la población masculina y a la pareja infértil, para ser casi nula cuando se indagó sobre la familia. Lo anterior puede tener una explicación que está sustentada en la revisión de resultados de investigaciones realizadas en el contexto cubano y en la observación, hasta el momento empírica, de los servicios de atención a la infertilidad.

Los argumentos parten desde las propias características de la atención a la infertilidad con énfasis en las causas biológicas del problema de salud, por lo que se concibe aún como una anormalidad que debe ser resuelta, como un error que debe subsanarse. ${ }^{10}$ Se atiende la infertilidad, no a personas que viven y padecen un problema de salud. Dicha atención está además regulada por un protocolo de atención que trata de estandarizar prácticas, diagnósticos y tratamientos, pero que excluye la exploración de la vivencia y el padecer de la pareja, lo que además se ve reforzado por los puntos de encuentro según la perspectiva de género de prestadores y usuarios de los servicios.

Aun cuando según protocolo debe exigirse la concurrencia en pareja a los servicios de atención a la infertilidad, persiste la morosidad de los varones, y cuando asisten, es la mujer quien posee historia clínica a los efectos de la institución de salud y quien recibe las indicaciones para las pruebas que deben realizarse sus parejas. Todos ello justifica en buena parte, la poca información que posee la mayoría de los entrevistados en cuanto a la vivencia del padecer para hombres, para la pareja infértil y la familia cubana.

La paternidad y la maternidad, desde la perspectiva antropológica, debe ser entendida no como un hecho natural, sino como una construcción sociocultural que se encuentra al centro del debate entorno a las consecuencias de la fuerte dicotomía que existe en muchas sociedades, entre la masculinidad (asociada a la función de proveedor económico) y la feminidad (destinada al cuidado diario de los hijos). ${ }^{11}$ Es ya un dato aportado por investigaciones precedentes, que la paternidad y la maternidad tienen significados diferentes para las personas de acuerdo no sólo con su sexo, ${ }^{3}$ sino también según otras variables socioculturales. 
Más allá de la reproducción biológica, son la reproducción social y los espacios donde esta se desarrolla, las que determinan en última instancia las percepciones individuales y colectivas de la función reproductiva y la infertilidad.

\section{REFERENCIAS BIBLIOGRÁFICAS}

1. Moreno Rosset C. Ansiedad y depresión: principales trastornos asociados a la infertilidad. I Congreso Virtual de Psiquiatría, 1 de Febrero-15 de Marzo 2000. Conferencia 28-CI-F [sitio en Internet]. [citado May 2009]. Disponible en: http://www.psiquiatria.com/congreso old/mesas/mesa28/conferencias/28 ci f.htm

2. Calero JL, Santana F. La infertilidad como evento de frustración personal. Reflexiones de un grupo de varones de parejas infértiles. Rev Cubana Endocrinol. 2006;17(1).

3. Olavarría J. Ser padre en Santiago. En: Fuller N, editor. Paternidades en América Latina. Lima: Fondo Editorial de la Pontificia Universidad Católica del Perú; 2000.

4. Callejo Gallego J. Observación, entrevista y grupo de discusión: El silencio de tres prácticas de investigación. Rev Esp Salud Pública. 2002;76:4009-422.

5. Acuña VH. La historia oral, las historias de vida y las ciencias sociales. Historia: teoría y métodos. San José: EDUCA; 1988.

6. Piña C. La construcción de "sí mismo" en el relato autobiográfico. Revista Paraguaya de Sociología. 1988;Año 25(71):135-76.

7. Vargas LA, Casillas LE. Perspectiva antropológica de la participación comunitaria para la promoción de la salud. En: García Viveros M, editor. Salud comunitaria y promoción de la salud. Las Palmas de Gran Canaria: ICEPSS; 1999. p.131-45.

8. Declaración de IMAP sobre la infertilidad. Bol Med IPPF. 1995;29(1).

9. Hurtado F. Salud sexual. Bases para una sexualidad saludable. Influencia de la anticoncepción y de la infertilidad. Revista Iberoamericana de Fertilidad. VI Congreso SEC. Marzo, 2002.

10. Castañeda $\mathrm{E}$, Bustos $\mathrm{HH}$. La ruta del padecer en mujeres con diagnóstico de infertilidad. Perinatol Reprod Hum. 2001;15(2):124-32.

11. Fuller N. Masculinidades. Cambios y permanencias. Lima: Fondo Editorial de la Pontificia Universidad Católica del Perú; 2001.

*Código del entrevistado/a.

Recibido: 5 de octubre de 2009.

Aprobado: 4 de febrero de 2010. 
Zoe Díaz Bernal. Escuela Nacional de Salud Pública. Calle Línea esq. a I. El Vedado 10400. La Habana, Cuba.

Teléf.: 641-8447,830-2533. E-mail: zoe@ensap.sld.cu, dailys@fbio.uh.cu 